\title{
Cardiovascular outcomes associated with use of clarithromycin: population based study
}

\author{
Angel Y S Wong, ${ }^{1}$ Adrian Root, ${ }^{2}$ Ian J Douglas, ${ }^{2}$ Celine S L Chui,, ${ }^{1}$ Esther W Chan, ${ }^{1}$ \\ Yonas Ghebremichael-Weldeselassie, ${ }^{3}$ Chung-Wah Siu, ${ }^{4}$ Liam Smeeth, ${ }^{2}$ Ian C K Wong ${ }^{1,5}$
}

${ }^{1}$ Centre for Safe Medication Practice and Research,

Department of Pharmacology and Pharmacy, Li Ka Shing Faculty of Medicine, University of Hong Kong, Hong Kong, China

2Faculty of Epidemiology and Population Health, London School of Hygiene and Tropical Medicine, London, UK

${ }^{3}$ Department of Mathematics and Statistics, Open University, Milton Keynes, UK

${ }^{4}$ Cardiology Division,

Department of Medicine, Li Ka Shing Faculty of Medicine,

University of Hong Kong, Hong

Kong, China

${ }^{5}$ Research Department of Practice and Policy, UCL School of Pharmacy, London, UK

Correspondence to: I C K Wong, Research Department of Practice and Policy, UCL School of Pharmacy, London WC1N 1AX,UKi.wong@ucl.ac.uk Additional material is published online only. To view please visit the journal online (http://dx.doi. org/10.1136/bmj.h6926)

Cite this as: $B M J$ 2016;352:h6926 http://dx.doi.org/10.1136/bmj.h6926 Accepted: 13 November 2015

\begin{abstract}
STUDY QUESTION

What is the association between clarithromycin use and cardiovascular outcomes?

\section{METHODS}

In this population based study the authors compared cardiovascular outcomes in adults aged 18 or more receiving oral clarithromycin or amoxicillin during 2005-09 in Hong Kong. Based on age within five years, sex, and calendar year at use, each clarithromycin user was matched to one or two amoxicillin users. The cohort analysis included patients who received clarithromycin ( $n=108988)$ or amoxicillin $(n=217793)$. The self controlled case series and case crossover analysis included those who received Helicobacter pylori eradication treatment containing clarithromycin. The primary outcome was myocardial infarction. Secondary outcomes were all cause, cardiac, or non-cardiac mortality, arrhythmia, and stroke.
\end{abstract}

\section{STUDY ANSWER AND LIMITATIONS}

The propensity score adjusted rate ratio of myocardial infarction 14 days after the start of antibiotic treatment was 3.66 (95\% confidence interval 2.82 to 4.76 ) comparing clarithromycin use (132 events, rate 44.4 per 1000 person years) with amoxicillin use (149 events, 19.2 per 1000 person years), but no long term increased risk was observed. Similarly, rate ratios of secondary outcomes increased significantly only with current use of clarithromycin versus amoxicillin, except for stroke. In the self controlled case analysis, there was an association between current use of $H$ pylori eradication treatment containing clarithromycin and cardiovascular events. The risk returned to baseline after treatment had ended. The case crossover analysis also showed an increased risk of cardiovascular events during current use of $H$ pylori eradication treatment containing clarithromycin. The adjusted absolute risk difference for current use of clarithromycin versus amoxicillin was

\section{WHAT IS ALREADY KNOWN ON THIS TOPIC}

Previous epidemiological studies suggest that clarithromycin is associated with an increased risk of serious cardiovascular outcomes

Whether the risk is short term or long term is unclear

\section{WHAT THIS STUDY ADDS}

In this population based study, current use of clarithromycin was associated with a significantly increased risk of myocardial infarction, arrhythmia, and cardiac death No long term effect was observed

The absolute risk for myocardial infarction and cardiac death was higher in those aged 75 or more or with hypertensive diseases or diabetes mellitus and therefore caution is needed when selecting antibiotics for these high risk patients

1.90 excess myocardial infarction events $(95 \%$ confidence interval 1.30 to 2.68 ) per 1000 patients.

WHAT THIS STUDY ADDS

Current use of clarithromycin was associated with an increased risk of myocardial infarction, arrhythmia, and cardiac mortality short term but no association with long term cardiovascular risks among the Hong Kong population.

FUNDING, COMPETING INTERESTS, DATA SHARING ID was funded by grants from the Medical Research Council for this project. LS was funded by a grant from the Wellcome Trust. The authors have no competing interests. No additional data are available.

\section{Introduction}

Clarithromycin is a commonly prescribed macrolide antibiotic, indicated for respiratory tract infections, Mycobacterium avium complex disease in patients with human immunodeficiency virus, and skin and soft tissue infections. ${ }^{12}$ Together with amoxicillin or metronidazole and a proton pump inhibitor, clarithromycin is also used to treat Helicobacter pylori infections. ${ }^{2}$ Several randomised controlled trials were conducted to explore the effects of clarithromycin on the prevention of cardiovascular outcomes among patients with cardiovascular disease. ${ }^{3-5}$ Among these, the first randomised controlled trial showed that clarithromycin use for three months seemed to reduce the risk of cardiovascular outcomes in 1.5 years. ${ }^{3}$ However, another randomised controlled trial, with follow-up of two years, showed that among patients receiving a coronary artery bypass graft, the rate of cardiovascular events was not significantly reduced with short term clarithromycin treatment compared with placebo. ${ }^{5}$ On the contrary, a significantly higher risk of cardiovascular mortality was reported over three years and also a higher all cause mortality among patients with coronary heart diseases receiving a two week course of clarithromycin once daily compared with placebo over six years. ${ }^{467}$

In addition to the randomised trials, a cohort study reported a substantially higher risk of cardiac death associated with use of clarithromycin compared with penicillin V over seven day's follow-up among the general population. ${ }^{8}$ Another cohort study also found a higher risk of cardiovascular events one year after treatment with clarithromycin compared with other antibiotics among patients with chronic obstructive pulmonary disease and community acquired pneumonia. ${ }^{9}$

Given the uncertainty of any increased cardiovascular risk with clarithromycin, and the finding of an effect well beyond the period of use, we carried out an observational study in Hong Kong to investigate whether 
clarithromycin is associated with cardiovascular outcomes among the general population. We conducted a cohort study using amoxicillin as comparator, a self controlled case series, and a case crossover study to eliminate between person confounding.

\section{Methods}

Data sources

The clinical data analysis and reporting system database is managed by the Hospital Authority, which is a statutory body responsible for primary, secondary, and tertiary public healthcare services in Hong Kong..$^{10}$ More than seven million Hong Kong residents have access to healthcare services provided by the Hospital Authority. The database contains information on patients' personal characteristics and includes details of diagnoses, operations, prescriptions, laboratory test results, visits to emergency departments and outpatient clinics, and hospital stay since 1993. It also contains data on causes of death through its internal linkage to regional deaths registry from the Immigration Department. The clinical data analysis and reporting system generated anonymous patient identifiers to protect patient confidentiality and to link all medical information such as diagnostic and prescribing data. This database has been used to conduct high quality epidemiological studies. ${ }^{11-13}$

\section{Cohort analysis}

We identified all adults aged 18 or more and prescribed either oral clarithromycin or oral amoxicillin (including amoxicillin with clavulanate potassium) between 1 January 2005 and 31 December 2009. Based on age within five years, sex, and calendar year at use, we matched one clarithromycin user with one or two amoxicillin users. In both groups we excluded patients who had been prescribed clarithromycin up to four years before the date of first antibiotic prescription during the observation period. However, amoxicillin users could be classified as using clarithromycin at a later date.

The observation period commenced from the date of the first antibiotic prescription (index date) and ended at the earliest occurrence of the outcome, death, subsequent use of clarithromycin or amoxicillin, or end of study (31 December 2012). We assumed that treatment was continuous if the gap between scripts was seven days or less, as the next script was likely to be prescribed for the same indication during follow-up consultation.

The primary outcome was first recorded myocardial infarction as the principal diagnosis for a visit to an emergency department or inpatient admission during the observation period in multiple follow-up periods. As the treatment duration of clarithromycin is generally seven to 14 days, we classified follow-up periods as current use (days 1-14 started on the index date), recent use (days 15-30), and past use (days 31-90, 91-365, 366-730, or 7311095). Secondary outcomes included first recorded principal diagnosis of arrhythmia; stroke; or all cause, cardiac, and non-cardiac mortality during the observation period. In each analysis we excluded patients with myocardial infarction, arrhythmia, or stroke before the observation period. We identified all outcomes using ICD-9 and ICD-10 codes (international classification of diseases, ninth and 10th revisions, see supplementary material 1).

\section{Self controlled case series analysis}

The self controlled case series method was developed to overcome confounding between participants by comparing the rate of outcomes in different periods within the same individual, and gives a relative incidence estimate. ${ }^{14}$ The method requires risk windows to be chosen a priori and is based on individuals who had both the drug of interest and the event. We adopted this caseonly approach to account for differences between participants. The exposure of interest was $H$ pylori eradication treatment containing clarithromycin. Acute infections are known to be associated with an increased risk of first myocardial infarction; for this reason we chose $H$ pylori because it is a chronic infection unlikely to be associated with a period of increased risk of myocardial infarction, and therefore unlikely to lead to a spurious association between clarithromycin and risk of myocardial infarction. ${ }^{15}$

We identified patients who received outpatient treatment for H pylori eradication of seven and 14 days' duration. Such treatment is defined as coprescription of clarithromycin with either amoxicillin or metronidazole together with one of the proton pump inhibitors, with doses as recommended by the British National Formulary (see supplementary material 2). To avoid including a selected group of patients with more severe health status at the prescription date, we included patients receiving outpatient treatment only. We excluded patients who received a clarithromycin prescription or inpatient treatment for $H$ pylori before outpatient treatment. Follow-up was censored if participants received a prescription for clarithromycin or inpatient treatment after outpatient treatment. Similar to the cohort analysis, the event outcomes included myocardial infarction, arrhythmia, and stroke. To be included in this cohort, patients required a first recorded event as principal diagnosis for a visit to an emergency department or inpatient admission during the study period (1 January 2003 to 31 December 2012).

The observation period started from one year after the patients entered the database, and we censored follow-up at the earliest of end of the study (31 December 2012), death, or one of the censoring events. We considered patients to be ineligible if they had a history of $H$ pylori eradication treatment or an event before the observation period. Similar to the cohort analysis, we defined several risk windows: current use (days 1-14 from the index date), recent use (days 15-30), and past use (days 31-90, 91-365, 366-730, or 731-1095). We classified all other periods as baseline, except for the 14 day window of risk before drug use. As the self controlled case series requires an assumption that an event should not alter the probability of subsequent drug use, we removed from the baseline periods a 14 day risk window before the use of $H$ pylori eradication treatment. This avoids the potential for bias whereby the outcome of interest (myocardial infarction, arrhythmia, stroke) may temporarily alter the likelihood of the drug being prescribed. ${ }^{14}$ When 
a gap in treatment was seven days or less we assumed treatment to have been continued. In addition to the standard self controlled case series, we also analysed the data using a non-parametric self controlled case series. This method, unlike that of the standard self controlled case series, does not require predefinition of several risk windows, and it estimates relative incidence functions showing how incidence changes with time. ${ }^{16}$

\section{Data validation}

To determine the validity of the recorded diagnoses, we reviewed the original clinical records in the computer based clinical management system among a sample of patients from the Hong Kong west cluster. In Hong Kong, all hospital admissions, outpatient clinic records, laboratory test results, and radiological images in the public health system have been recorded in the clinical management system since 1996. The Hong Kong west cluster, one of the seven administrative hospital clusters of the Hospital Authority, provides public healthcare services to 530000 residents ( $8 \%$ of the Hong Kong population) in that region and allows cross-cluster referrals from other cluster regions..$^{17}$ One of the two university teaching hospitals in the Hong Kong west cluster-the Queen Mary Hospital-provides tertiary and quaternary care. ${ }^{17}$

Through the clinical data analysis and reporting system we identified cases of myocardial infarction and arrhythmia among our study cohort. A cardiologist (CS) reviewed the records, including clinical notes, electrocardiograms, laboratory test results, and computed tomograms. We identified cases of stroke from another study cohort (WCY Lau, personal communication, 2015). The clinical notes and results from radiology, computed tomography, or magnetic resonance imaging of the brain were reviewed. We defined the corresponding positive predictive values with binominal exact 95\% confidence intervals as the proportion of the number of patients with verified outcomes after review against the total number of patients with the specific diagnosis in the clinical data analysis and reporting system.

\section{Statistical and sensitivity analyses}

After initial adjustment for age, sex, and history of the event of interest, we used Poisson regression to estimate the rate ratios for clarithromycin users compared with amoxicillin users during current, recent, and past use. To control for confounding we also adjusted for propensity score. We derived propensity scores from conditional logistic regression to represent the conditional probability of drug use given the covariates (see supplementary material 3). To identify a group within which valid comparisons were possible, we further restricted the cohort to those participants whose propensity scores were within the overlapping region of the distributions of the clarithromycin and amoxicillin groups. To further reduce the potential effects of unmeasured confounding, we excluded those with extreme scores in the upper or lower tail of the propensity score distribution to avoid bias owing to missing information on important risk factors for adverse outcomes. ${ }^{18}$ To allow establishment of the cut-points for trimming we therefore constructed 20 categories of $5 \%$ each for the distribution of scores. ${ }^{19}$ In the primary analysis we trimmed the first and 20th propensity score strata (see supplementary material 4). Along with other initial adjustment variables, we also used the estimated propensity score as an adjustment variable in the Poisson regression in the final analysis. We conducted a sensitivity analysis for follow-up periods in current (days 1-7 from the index date) and recent use (days 8-30). We also performed a sensitivity analysis without trimming propensity score strata. For the analyses of causes of death, patients with unknown cause of death at the time of death were censored but not classified as having an outcome. We also conducted a subgroup analysis to evaluate the risk of cardiovascular outcomes for age, sex, and history of hypertensive diseases or diabetes mellitus. Moreover, we estimated the propensity score adjusted absolute difference in risk of myocardial infarction, arrhythmia, stroke, and cardiac death for clarithromycin use as adjusted rate ratios minus 1, followed by the multiplication of the crude incidence rates among patients using amoxicillin for each 1000 patients. ${ }^{2021}$ Similarly, we estimated the propensity score adjusted absolute differences in subgroups. All statistical tests were two sided and we considered $P$ values at the $5 \%$ level to be statistically significant.

For the self controlled case series analysis, we estimated incidence rate ratios using conditional Poisson regression, comparing the rate of events during risk windows with the rate during baseline periods. We further divided all periods into single year bands to adjust for age effect. As myocardial infarction might increase mortality in the short term, the observation period would be censored at random and thus affect the assumption of event independence. As the self controlled case series analysis might be prone to bias if the outcome leads to censoring of the observation period, we also performed a post hoc case crossover analysis, which is not vulnerable to this limitation of the self controlled case series. The case crossover design is applied for studies investigating the association between transient drug use and outcome with abrupt time of onset. ${ }^{22}$ We estimated odds ratios using conditional logistic regression, comparing drug use before the event (current period) with that at other earlier control periods within patients. The length of current and control periods were both 14 days. We used 100 control periods at maximum to improve precision and increase power. ${ }^{22}$ As the temporal trend of prescriptions for $\mathrm{H}$ pylori eradication treatment containing clarithromycin was stable throughout the study period we did not adjust time trends in drug use for case crossover analysis (see supplementary material 5).

In the non-parametric self controlled case series analysis we estimated two incidence rate ratio functions, using myocardial infarction as an example to illustrate the incidence rate ratio over time; a function that represents an incidence rate ratio during current use of clarithromycin and an incidence rate ratio function in the washout period up to three years after treatment ended. In this method, age effect was also adjusted for without prespecifying age groups. 
For quality assurance, two investigators (AW and CC) independently conducted the statistical analyses using SAS software, version 9.3 (SAS Institute) and R version 3.2.0 (www.R-project.org).

\section{Results}

\section{Data validation}

A total of 1999 cases of myocardial infarction and 712 of arrhythmia were identified in the clinical data analysis and reporting system from cohort and self controlled case series analysis. Of these, we identified 151 cases of myocardial infarction and 44 of arrhythmia from the Hong Kong west cluster. In another study cohort, 1054 cases of stroke were identified in the analysis. Among these, 90 were from the Hong Kong west cluster. The overall positive predictive values for myocardial infarction (129/151), arrhythmia (20/44), and stroke (82/90) were 85.4\% (95\% confidence interval $78.8 \%$ to $90.6 \%$ ), $45.5 \%$ (30.4\% to $61.2 \%$ ), and $91.1 \%$ (83.2\% to $96.1 \%$ ), respectively.

\section{The risk of cardiovascular outcomes}

Figure 1 shows the flow of the cohort in the study. We matched 108988 clarithromycin users with 217793 amoxicillin users. Table 1 shows the baseline characteristics of the full cohort (see supplementary material 6 for characteristics of the trimmed cohort). Supplementary material 7 illustrates the propensity score distributions before and after trimming the propensity score. Compared with amoxicillin users, clarithromycin users were more likely to have a medical history of chronic obstructive pulmonary disease, obesity, hepatic problems, and heart failure. They were also more likely to have prescriptions for insulin, oral corticosteroids, proton pump inhibitors, and $\mathrm{H}_{2}$ receptor blockers, and to have visited an emergency department in the past year.

After exclusion of propensity score stratum 1 and 20, the cohort included 95797 clarithromycin users and 198305 amoxicillin users. Among these participants, 583 clarithromycin users and 1375 amoxicillin users had the primary outcome of myocardial infarction during follow-up.

Figure 2 and table 2 show the adjusted rate ratios of all outcomes associated with current, recent, and past use of clarithromycin compared with use of amoxicillin.

\section{Patients receiving clarithromycin ( $n=139770)$}

Excluded $(n=30780)$

Parental dose forms $(n=2698)$

Clarithromycin prescription before

index date $(\mathrm{n}=9432)$

Invalid demographics or age $<18$ years

$(n=15$ 572)

Incomplete records $(\mathrm{n}=3078)$
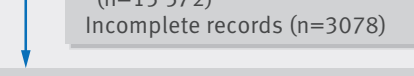

Fig 1 | Inclusion and exclusion of participants in cohort analysis
For myocardial infarction, the initial adjusted rate ratio for current use of clarithromycin was 2.72 (95\% confidence interval 2.15 to 3.44 ), with a propensity score adjusted rate ratio of 3.66 (2.82 to 4.76) compared with use of amoxicillin. For the secondary outcomes of arrhythmia and stroke, the initial adjusted rate ratios for current use of clarithromycin were 2.13 (1.25 to 3.63) and 1.05 (0.79 to 1.41), respectively, with fully propensity score adjusted rate ratios of 2.22 (1.22 to 4.06) and 1.11 (0.80 to 1.54) compared with current use of amoxicillin. For all cause mortality, the initial adjusted rate ratio for current use of clarithromycin was 2.41 (2.27 to 2.56), with a propensity score adjusted rate ratio of 1.97 (1.83 to 2.11) compared with current use of amoxicillin. For cardiac mortality, the initial rate ratio for use of clarithromycin was 1.93 (1.61 to 2.30), with a propensity score adjusted rate ratio of 1.67 (1.36 to 2.06) compared with current use of amoxicillin. For non-cardiac mortality, the initial adjusted rate ratio associated with current use of clarithromycin was 2.59 (2.42 to 2.76), with propensity score adjusted rate ratio of 2.10 (1.94 to 2.27). No statistically significant increased rate ratios of all outcomes were found for recent and past use of clarithromycin compared with recent and past use of amoxicillin. In the sensitivity analysis when we included patients with scores in the first and 20th 5\% stratums, similar results were obtained for all outcomes of interest in the primary analysis (see supplementary material 8).

Similar results were obtained for all outcomes of interest in the primary analysis when the sensitivity analysis was conducted, stratifying follow-up periods for current use (days 1-7) and recent use (days 8-30) of clarithromycin compared with amoxicillin (see supplementary material 9). The increased cardiovascular risk was largely confined to current use (days 1-7) of clarithromycin versus amoxicillin. Subgroup analysis showed that rate ratios for myocardial infarction, arrhythmia, and cardiac death were comparable between men and women. The highest absolute risk differences were for myocardial infarction and cardiac death in patients aged 75 or more and for those with a history of hypertensive diseases or diabetes. Owing to the lack of power, evidence was lacking for an increased risk of arrhythmia among patients aged 65 or more and those with a history of hypertensive diseases or diabetes (see supplementary material 10). The propensity score adjusted absolute risk difference for current use of clarithromycin compared with amoxicillin was 1.90 excess myocardial infarction events (95\% confidence interval 1.30 to 2.68 ) per 1000 patients. Also, 0.95 excess cardiac deaths ( 0.51 to 1.51 ) and 0.20 excess arrhythmia events (0.04 to 0.49$)$ per 1000 patients occurred in current users of clarithromycin compared with current users of amoxicillin.

In the self controlled case series analysis, for those patients who received outpatient treatment for eradication of $H$ pylori we identified 740 patients who had incident myocardial infarction during the study period, 309 who had incident arrhythmia, and 2279 who had incident stroke. Table 3 presents the incidence rate ratios 
Table 1 | Baseline characteristics of full cohort at index date in cohort analysis. Values are numbers (percentages) unless stated otherwise

\begin{tabular}{|c|c|c|}
\hline Characteristics & $\begin{array}{l}\text { Clarithromycin users } \\
(\mathrm{n}=108988)\end{array}$ & $\begin{array}{l}\text { Amoxicillin users } \\
(\mathrm{n}=217793)\end{array}$ \\
\hline Median age (years) & 60.0 & 60.0 \\
\hline Men & $57114(52.4)$ & $114063(52.4)$ \\
\hline \multicolumn{3}{|l|}{ Calendar month of drug use: } \\
\hline January & $9387(8.6)$ & $19950(9.2)$ \\
\hline February & $9046(8.3)$ & $18512(8.5)$ \\
\hline March & $10163(9.3)$ & $20961(9.6)$ \\
\hline April & $9387(8.6)$ & $18928(8.7)$ \\
\hline May & $9353(8.6)$ & $18992(8.7)$ \\
\hline June & $8919(8.2)$ & $18219(8.4)$ \\
\hline July & $9077(8.3)$ & $18006(8.3)$ \\
\hline August & $9080(8.3)$ & $17622(8.1)$ \\
\hline September & $8539(7.8)$ & $16858(7.7)$ \\
\hline October & $8517(7.8)$ & $16370(7.5)$ \\
\hline November & $8541(7.8)$ & $16289(7.5)$ \\
\hline December & $8979(8.2)$ & $17086(7.9)$ \\
\hline \multicolumn{3}{|l|}{ Medical history: } \\
\hline Chronic obstructive pulmonary disease & $7528(6.9)$ & $6946(3.2)$ \\
\hline Obesity & $339(0.3)$ & $399(0.2)$ \\
\hline Diabetes & $10761(9.9)$ & $16287(7.5)$ \\
\hline Thyroid disorders & $1813(1.7)$ & $3457(1.6)$ \\
\hline Hyperlipidaemia & $4348(4.0)$ & $6749(3.1)$ \\
\hline Hypertensive diseases & $17788(16.3)$ & $28067(12.9)$ \\
\hline Coronary heart disease & $8200(7.5)$ & $12883(5.9)$ \\
\hline Cerebrovascular diseases & $8696(8.0)$ & $13032(6.0)$ \\
\hline Arterial disease & $2132(2.0)$ & $2970(1.4)$ \\
\hline Cardiomyopathy & $382(0.4)$ & $545(0.3)$ \\
\hline Heart failure & $5707(5.2)$ & $6791(3.1)$ \\
\hline Valve disorders & $663(0.6)$ & $1211(0.6)$ \\
\hline Arrhythmia and conduction disorders & $6359(5.8)$ & $10404(4.8)$ \\
\hline Hypertensive renal disease & $404(0.4)$ & $475(0.2)$ \\
\hline Renal failure & $2675(2.5)$ & $3146(1.4)$ \\
\hline Oesophageal varices & $274(0.3)$ & $192(0.1)$ \\
\hline Hepatic failure, liver fibrosis, and cirrhosis & $1572(1.4)$ & $1764(0.8)$ \\
\hline Schizophrenia, delusional disorders, and psychosis & $2114(1.9)$ & $4286(2.0)$ \\
\hline Bipolar disorder & $226(0.2)$ & $488(0.2)$ \\
\hline Depression & $3096(2.8)$ & $5386(2.5)$ \\
\hline \multicolumn{3}{|l|}{ Prescription in past year: } \\
\hline Angiotensin receptor blocker/angiotensin converting enzyme inhibitor & $16573(15.2)$ & $28559(13.1)$ \\
\hline Calcium channel blockers & $22098(20.3)$ & $40597(18.6)$ \\
\hline Loop diuretics & $10137(9.3)$ & $12091(5.6)$ \\
\hline Other diuretics & $8989(8.3)$ & $17691(8.1)$ \\
\hline$\beta$ blockers & $19295(17.7)$ & $36691(16.9)$ \\
\hline Anti-arrhythmics class I and II & $1217(1.1)$ & $1677(0.8)$ \\
\hline Digoxin & $2737(2.5)$ & $3760(1.7)$ \\
\hline Nitrates & $9932(9.1)$ & $14492(6.7)$ \\
\hline Platelet inhibitors & $17917(16.4)$ & $28159(12.9)$ \\
\hline Anticoagulants & $3768(3.5)$ & $5830(2.7)$ \\
\hline Peripheral vasodilators & $974(0.9)$ & $1455(0.7)$ \\
\hline Lipid lowering drugs & $9585(8.8)$ & $16979(7.8)$ \\
\hline Insulin & $3737(3.4)$ & $4228(1.9)$ \\
\hline Oral hypoglycaemic agent & $13080(12.0)$ & $23720(10.9)$ \\
\hline Antidepressants & $5536(5.1)$ & $9359(4.3)$ \\
\hline Antipsychotics & $4508(4.1)$ & $7559(3.5)$ \\
\hline Oral corticosteroids & $9945(9.1)$ & $9600(4.4)$ \\
\hline Non-steroidal anti-inflammatory drugs & $18856(17.3)$ & $40139(18.4)$ \\
\hline Proton pump inhibitors $/ \mathrm{H}_{2}$ receptor blockers & $55615(51.0)$ & $44190(20.3)$ \\
\hline Median No (interquartile range) of prescriptions used in past year & $17(4-44)$ & $12(2-31)$ \\
\hline \multicolumn{3}{|l|}{ Healthcare use in past year: } \\
\hline Median No (interquartile range) of outpatient visits & $5(2-10)$ & $5(1-9)$ \\
\hline Median No (interquartile range) of inpatient stays & $1(0-2)$ & $0(0-1)$ \\
\hline Median No (interquartile range) of emergency department visits & $1(0-2)$ & $0(0-1)$ \\
\hline
\end{tabular}




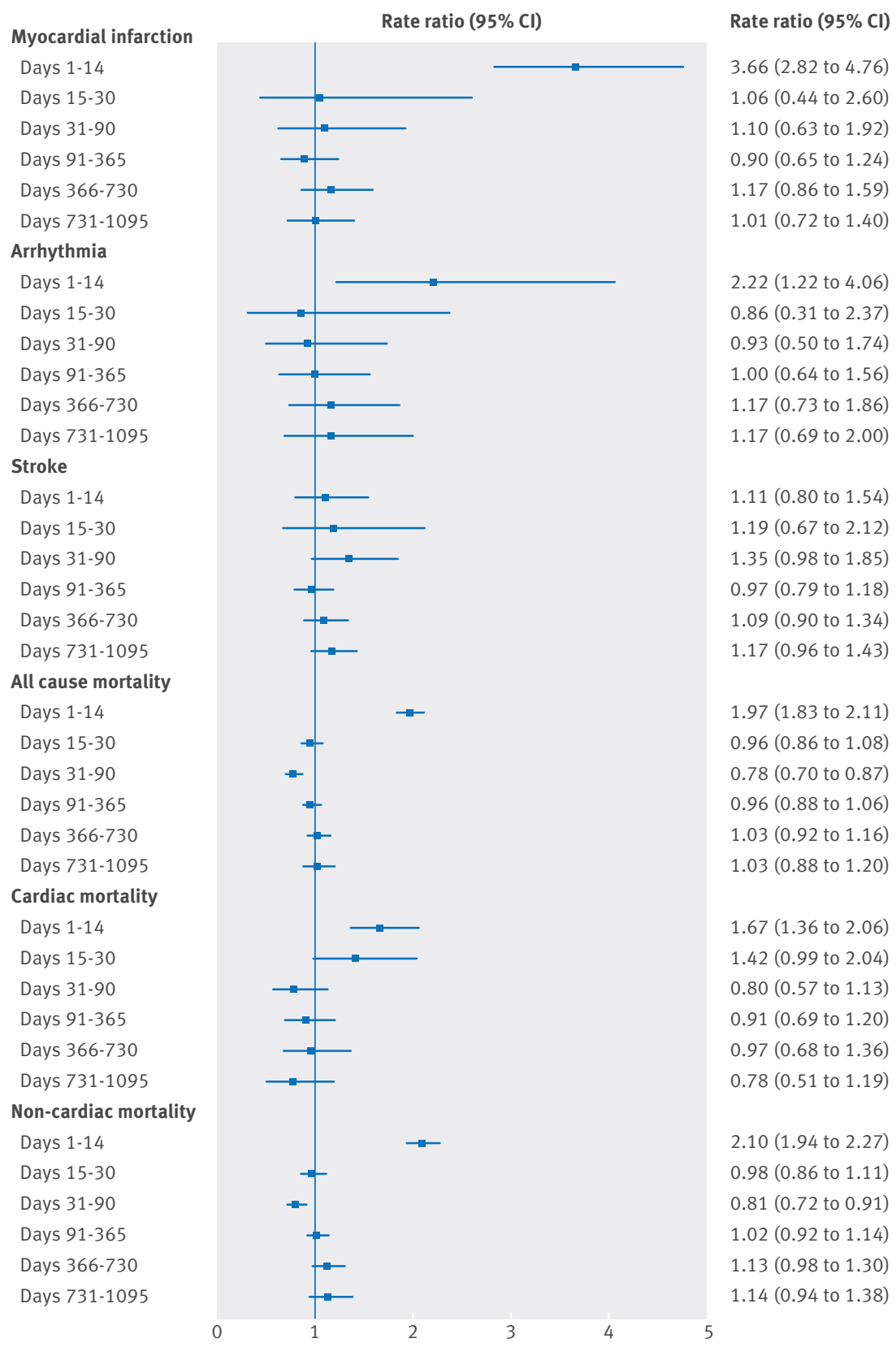

Fig 2 | Propensity score adjusted rate ratios of all outcomes with clarithromycin use compared with amoxicillin use
14 days of current $H$ pylori eradication treatment. The risk of myocardial infarction was increased during this period and the risk reduced to a baseline rate around the end of treatment. The incidence rate ratio of myocardial infarction after $H$ pylori eradication treatment did not change over time, but rather stayed at the baseline rate (fig 3 ).

\section{Discussion}

In this territory wide cohort study, no increased risk of cardiovascular outcomes was observed long term with clarithromycin treatment compared with amoxicillin treatment but there was a suggestion of an increased cardiovascular risk during and immediately after clarithromycin use. A case only approach was employed to investigate the association between clarithromycin as part of $H$ pylori eradication treatment and cardiovascular outcomes. Similarly, we observed that the risk of myocardial infarction and arrhythmia was only increased during current use of $H$ pylori eradication treatment containing clarithromycin, and no increased risk was observed long term.

\section{Interpretation and comparison with other studies}

In an attempt to minimise indication bias in the cohort analysis, we selected amoxicillin as the comparator group, as it is used for similar indications to clarithromycin. However, the characteristics of the clarithromycin users at the index date were systematically different from those of amoxicillin users-a higher proportion of those taking clarithromycin had chronic obstructive pulmonary disease, diabetes, hepatic problems, coronary heart disease, and heart failure. These patients were also more likely to use a proton pump inhibitor, which has been identified as a marker of poor prognosis in previous work. ${ }^{23}$ It is possible that clinicians opt for clarithromycin in frailer patients. Although the presence of channeling bias seems to be implied, the underlying differences in health between the treatment groups did not appear to fully explain the noticeably increased short term cardiovascular risk with clarithromycin use based on the propensity score adjustment method.

A higher rate ratio of non-cardiac mortality was also found in the cohort analysis for current use of clarithromycin compared with amoxicillin despite adjustment for propensity score, which would not be predicted by the study hypothesis. As a negative control outcome, this may indicate bias. This further suggests that, compared with amoxicillin, clarithromycin was more likely to be prescribed for frailer patients or for those with more severe infections. However, it is anticipated that all between person confounding factors that remain stable in a cohort study design could be removed over the study period by using self controlled case series methodology. Using this analysis method and focusing on patients treated for $H$ pylori infection, which is not an acute infection, we still found an increased risk of myocardial infarction and arrhythmia during current use of $H$ pylori eradication treatment containing clarithromycin, with no increased risk observed after treatment had ended. 


\begin{tabular}{|c|c|c|c|c|c|c|c|}
\hline \multirow[b]{2}{*}{$\begin{array}{l}\text { Outcomes by } \\
\text { treatment duration }\end{array}$} & \multicolumn{4}{|c|}{ Before propensity score adjustment } & \multicolumn{3}{|c|}{$\begin{array}{l}\text { Analysis restricted to patients with } \\
\text { comparable propensity scores }\end{array}$} \\
\hline & $\begin{array}{l}\text { No of } \\
\text { patients }\end{array}$ & $\begin{array}{l}\text { Mean follow-up } \\
\text { (days) }\end{array}$ & $\begin{array}{l}\text { No of } \\
\text { events }\end{array}$ & Rate ratio $(95 \% \mathrm{Cl})$ & $\begin{array}{l}\text { No of } \\
\text { patients }\end{array}$ & $\begin{array}{l}\text { No of } \\
\text { events }\end{array}$ & Rate ratio $(95 \% \mathrm{Cl})$ \\
\hline \multicolumn{8}{|l|}{ Myocardial infarction } \\
\hline \multicolumn{8}{|c|}{ Days 1-14: } \\
\hline Clarithromycin & 102514 & 10.6 & 132 & $2.72(2.15$ to 3.44$)$ & 90411 & 117 & $3.66(2.82$ to 4.76$)$ \\
\hline Amoxicillin & 204855 & 13.9 & 149 & - & 186888 & 132 & - \\
\hline \multicolumn{8}{|l|}{ Days 15-30: } \\
\hline Clarithromycin & 70184 & 15.7 & 11 & $1.23(0.62$ to 2.43$)$ & 62803 & 7 & $1.06(0.44$ to 2.60$)$ \\
\hline Amoxicillin & 200770 & 15.7 & 34 & - & 183379 & 25 & - \\
\hline \multicolumn{8}{|l|}{ Days 31-90: } \\
\hline Clarithromycin & 67707 & 57.8 & 23 & 1.31 (0.82 to 2.12 ) & 60826 & 19 & 1.10 (0.63 to 1.92$)$ \\
\hline Amoxicillin & 193522 & 57.7 & 67 & - & 177022 & 59 & - \\
\hline \multicolumn{8}{|l|}{ Days 91-365: } \\
\hline Clarithromycin & 63155 & 251.4 & 67 & 1.15 (0.87 to 1.52$)$ & 57197 & 56 & 0.90 (0.65 to 1.24$)$ \\
\hline Amoxicillin & 180522 & 252.2 & 221 & - & 165594 & 198 & - \\
\hline \multicolumn{8}{|l|}{ Days 366-730: } \\
\hline Clarithromycin & 53614 & 339.8 & 72 & 1.23 (0.94 to 1.61$)$ & 49126 & 64 & 1.17 (0.86 to 1.59$)$ \\
\hline Amoxicillin & 153426 & 336.2 & 223 & - & 141460 & 205 & - \\
\hline \multicolumn{8}{|l|}{ Days 731-1095: } \\
\hline Clarithromycin & 46586 & 344.4 & 62 & 1.18 (0.88 to 1.57$)$ & 42977 & 57 & 1.01 (0.72 to 1.40$)$ \\
\hline Amoxicillin & 130664 & 340.7 & 198 & - & 120937 & 182 & - \\
\hline \multicolumn{8}{|l|}{ Arrhythmia } \\
\hline \multicolumn{8}{|l|}{ Days 1-14: } \\
\hline Clarithromycin & 107200 & 10.5 & 23 & $2.13(1.25$ to 3.63$)$ & 94319 & 20 & $2.22(1.22$ to 4.06$)$ \\
\hline Amoxicillin & 214222 & 13.9 & 33 & - & 195129 & 31 & - \\
\hline \multicolumn{8}{|l|}{ Days 15-30: } \\
\hline Clarithromycin & 72574 & 15.7 & 7 & 0.97 (0.42 to 2.23$)$ & 64826 & 5 & 0.86 (0.31 to 2.37$)$ \\
\hline Amoxicillin & 209867 & 15.7 & 27 & - & 191386 & 24 & - \\
\hline \multicolumn{8}{|l|}{ Days 31-90: } \\
\hline Clarithromycin & 69933 & 57.7 & 17 & 0.98 (0.58 to 1.68$)$ & 62723 & 14 & 0.93 (0.50 to 1.74$)$ \\
\hline Amoxicillin & 202075 & 57.7 & 66 & - & 184556 & 59 & - \\
\hline \multicolumn{8}{|l|}{ Days 91-365: } \\
\hline Clarithromycin & 65072 & 250.9 & 41 & 1.42 (0.99 to 2.04$)$ & 58854 & 30 & $1.00(0.64$ to 1.56$)$ \\
\hline Amoxicillin & 188113 & 252.0 & 110 & - & 172294 & 97 & - \\
\hline Days 366-730: & & & & & & & \\
\hline Clarithromycin & 55081 & 339.6 & 36 & 1.28 (0.87 to 1.88$)$ & 50425 & 28 & $1.17(0.73$ to 1.86$)$ \\
\hline Amoxicillin & 159549 & 335.8 & 100 & - & 146877 & 88 & - \\
\hline Days 731-1095: & & & & & & & \\
\hline Clarithromycin & 47788 & 344.2 & 28 & $1.23(0.80$ to 1.91$)$ & 44042 & 22 & 1.17 (0.69 to 2.00$)$ \\
\hline Amoxicillin & 135506 & 340.4 & 81 & - & 125218 & 69 & - \\
\hline Stroke & & & & & & & \\
\hline Days 1-14: & & & & & & & \\
\hline Clarithromycin & 90225 & 10.8 & 63 & 1.05 (0.79 to 1.41$)$ & 80236 & 54 & 1.11 (0.80 to 1.54$)$ \\
\hline Amoxicillin & 180324 & 13.9 & 176 & - & 164634 & 163 & - \\
\hline Days 15-30: & & & & & & & \\
\hline Clarithromycin & 63829 & 15.7 & 21 & 1.20 (0.73 to 1.97$)$ & 57412 & 18 & 1.19 (0.67 to 2.12 ) \\
\hline Amoxicillin & 177173 & 15.7 & 61 & - & 161908 & 55 & - \\
\hline Days 31-90: & & & & & & & \\
\hline Clarithromycin & 61804 & 58.0 & 79 & 1.57 (1.20 to 2.05$)$ & 55768 & 64 & 1.35 (0.98 to 1.85$)$ \\
\hline Amoxicillin & 171158 & 57.9 & 176 & - & 156616 & 159 & - \\
\hline Days 91-365: & & & & & & & \\
\hline Clarithromycin & 57983 & 252.4 & 178 & $1.09(0.92$ to 1.29$)$ & 52658 & 151 & 0.97 (0.79 to 1.18$)$ \\
\hline Amoxicillin & 160206 & 253.2 & 573 & - & 146937 & 521 & - \\
\hline Days 366-730: & & & & & & & \\
\hline Clarithromycin & 49616 & 341.0 & 177 & $1.12(0.95$ to 1.33$)$ & 45525 & 154 & 1.09 (0.90 to 1.34$)$ \\
\hline Amoxicillin & 137150 & 337.6 & 555 & - & 126440 & 505 & - \\
\hline Days 731-1095: & & & & & & & \\
\hline Clarithromycin & 43398 & 345.2 & 172 & 1.14 (0.96 to 1.36$)$ & 40064 & 154 & 1.17 (0.96 to 1.43$)$ \\
\hline Amoxicillin & 117679 & 341.7 & 524 & - & 108918 & 473 & - \\
\hline & & & & & & & (Continued) \\
\hline
\end{tabular}




\begin{tabular}{|c|c|c|c|c|c|c|c|}
\hline \multirow[b]{2}{*}{$\begin{array}{l}\text { Outcomes by } \\
\text { treatment duration }\end{array}$} & \multicolumn{4}{|c|}{ Before propensity score adjustment } & \multicolumn{3}{|c|}{$\begin{array}{l}\text { Analysis restricted to patients with } \\
\text { comparable propensity scores }\end{array}$} \\
\hline & $\begin{array}{l}\text { No of } \\
\text { patients }\end{array}$ & $\begin{array}{l}\text { Mean follow-up } \\
\text { (days) }\end{array}$ & $\begin{array}{l}\text { No of } \\
\text { events }\end{array}$ & Rate ratio $(95 \% \mathrm{Cl})$ & $\begin{array}{l}\text { No of } \\
\text { patients }\end{array}$ & $\begin{array}{l}\text { No of } \\
\text { events }\end{array}$ & Rate ratio $(95 \% \mathrm{Cl})$ \\
\hline \multicolumn{8}{|l|}{ All cause mortality } \\
\hline \multicolumn{8}{|l|}{ Days 1-14: } \\
\hline Clarithromycin & 108988 & 10.5 & 1948 & $2.41(2.27$ to 2.56$)$ & 95797 & 1471 & 1.97 (1.83 to 2.11$)$ \\
\hline Amoxicillin & 217793 & 13.9 & 2562 & - & 198305 & 2127 & - \\
\hline \multicolumn{8}{|l|}{ Days 15-30: } \\
\hline Clarithromycin & 73526 & 15.7 & 569 & 1.36 (1.24 to 1.50$)$ & 65641 & 413 & 0.96 (0.86 to 1.08$)$ \\
\hline Amoxicillin & 213280 & 15.7 & 1611 & - & 194421 & 1352 & - \\
\hline \multicolumn{8}{|l|}{ Days 31-90: } \\
\hline Clarithromycin & 70820 & 57.7 & 659 & $1.11(1.02$ to 1.21$)$ & 63492 & 506 & $0.78(0.70$ to 0.87$)$ \\
\hline Amoxicillin & 205318 & 57.7 & 2283 & - & 187445 & 1920 & - \\
\hline \multicolumn{8}{|l|}{ Days 91-365: } \\
\hline Clarithromycin & 65875 & 250.9 & 836 & $1.28(1.18$ to 1.39$)$ & 59563 & 669 & 0.96 (0.88 to 1.06$)$ \\
\hline Amoxicillin & 191071 & 251.9 & 2563 & - & 174938 & 2251 & - \\
\hline \multicolumn{8}{|l|}{ Days 366-730: } \\
\hline Clarithromycin & 55750 & 339.4 & 505 & $1.30(1.18$ to 1.44$)$ & 51023 & 418 & $1.03(0.92$ to 1.16$)$ \\
\hline Amoxicillin & 161952 & 335.8 & 1544 & - & 149045 & 1392 & - \\
\hline \multicolumn{8}{|l|}{ Days 731-1095: } \\
\hline Clarithromycin & 48329 & 344.1 & 283 & 1.20 (1.05 to 1.38$)$ & 44534 & 241 & $1.03(0.88$ to 1.20$)$ \\
\hline Amoxicillin & 137515 & 340.3 & 954 & - & 127043 & 853 & - \\
\hline \multicolumn{8}{|l|}{ Cardiac mortality $\neq \S$} \\
\hline \multicolumn{8}{|l|}{ Days 1-14: } \\
\hline Clarithromycin & 108988 & 10.5 & 201 & 1.93 (1.61 to 2.30$)$ & 95797 & 152 & $1.67(1.36$ to 2.06$)$ \\
\hline Amoxicillin & 217793 & 13.9 & 315 & - & 198305 & 279 & - \\
\hline \multicolumn{8}{|l|}{ Days 15-30: } \\
\hline Clarithromycin & 73526 & 15.7 & 55 & $1.46(1.07$ to 1.99$)$ & 65641 & 43 & 1.42 (0.99 to 2.04$)$ \\
\hline Amoxicillin & 213280 & 15.7 & 152 & - & 194421 & 130 & - \\
\hline \multicolumn{8}{|l|}{ Days 31-90: } \\
\hline Clarithromycin & 70820 & 57.7 & 54 & $1.01(0.75$ to 1.36$)$ & 63492 & 43 & 0.80 (0.57 to 1.13$)$ \\
\hline Amoxicillin & 205318 & 57.7 & 219 & - & 187445 & 194 & - \\
\hline \multicolumn{8}{|l|}{ Days 91-365: } \\
\hline Clarithromycin & 65875 & 250.9 & 84 & $1.15(0.90$ to 1.46$)$ & 59563 & 70 & 0.91 (0.69 to 1.20$)$ \\
\hline Amoxicillin & 191071 & 251.9 & 313 & - & 174938 & 273 & - \\
\hline \multicolumn{8}{|l|}{ Days 366-730: } \\
\hline Clarithromycin & 55750 & 339.4 & 53 & 1.10 (0.81 to 1.49$)$ & 51023 & 46 & 0.97 (0.68 to 1.36$)$ \\
\hline Amoxicillin & 161952 & 335.8 & 210 & - & 149045 & 189 & - \\
\hline Days 731-1095: & & & & & & & \\
\hline Clarithromycin & 48329 & 344.1 & 35 & 0.94 (0.65 to 1.36$)$ & 44534 & 29 & 0.78 (0.51 to 1.19$)$ \\
\hline Amoxicillin & 137515 & 340.3 & 160 & - & 127043 & 144 & - \\
\hline Non-cardiac mortal & & & & & & & \\
\hline Days 1-14: & & & & & & & \\
\hline Clarithromycin & 108988 & 10.5 & 1687 & 2.59 (2.42 to 2.76$)$ & 95797 & 1271 & 2.10 (1.94 to 2.27$)$ \\
\hline Amoxicillin & 217793 & 13.9 & 2067 & - & 198305 & 1695 & - \\
\hline Days 15-30: & & & & & & & \\
\hline Clarithromycin & 73526 & 15.7 & 490 & $1.40(1.26$ to 1.56$)$ & 65641 & 357 & 0.98 (0.86 to 1.11$)$ \\
\hline Amoxicillin & 213280 & 15.7 & 1335 & - & 194421 & 1112 & - \\
\hline Days 31-90: & & & & & & & \\
\hline Clarithromycin & 70820 & 57.7 & 555 & 1.16 (1.05 to 1.28$)$ & 63492 & 424 & 0.81 (0.72 to 0.91$)$ \\
\hline Amoxicillin & 205318 & 57.7 & 1808 & - & 187445 & 1492 & - \\
\hline Days 91-365: & & & & & & & \\
\hline Clarithromycin & 65875 & 250.9 & 655 & 1.39 (1.27 to 1.53$)$ & 59563 & 517 & 1.02 (0.92 to 1.14$)$ \\
\hline Amoxicillin & 191071 & 251.9 & 1799 & - & 174938 & 1561 & - \\
\hline Days 366-730: & & & & & & & \\
\hline Clarithromycin & 55750 & 339.4 & 387 & 1.43 (1.27 to 1.61$)$ & 51023 & 318 & 1.13 (0.98 to 1.30$)$ \\
\hline Amoxicillin & 161952 & 335.8 & 1052 & - & 149045 & 943 & - \\
\hline Days 731-1095: & & & & & & & \\
\hline Clarithromycin & 48329 & 344.1 & 195 & 1.30 (1.11 to 1.53$)$ & 44534 & 168 & 1.14 (0.94 to 1.38$)$ \\
\hline Amoxicillin & 137515 & 340.3 & 592 & - & 127043 & 532 & - \\
\hline
\end{tabular}

Days 1-14=current use; days 15-30=recent use; days 31-1095=past use.

* Initial adjustment for age and sex before propensity score adjustment.

\#Initial adjustment for age, sex, and history of myocardial infarction and arrhythmia before propensity score adjustment.

tFull adjustment for age, sex, and propensity score in analyses restricted to patients with comparable propensity scores.

§Full adjustment for age, sex, history of myocardial infarction and arrhythmia, and propensity score in analyses restricted to patients with comparable propensity scores. 


\begin{tabular}{|c|c|c|}
\hline Characteristics & Self controlled case series & Case crossover \\
\hline \multicolumn{3}{|l|}{ Myocardial infarction } \\
\hline No in sample & 740 & 308 \\
\hline Median age (years)* & 65.0 & 73.3 \\
\hline Men (\%) & $508(68.7)$ & $201(65.3)$ \\
\hline 14 days before treatment & 0.81 (0.26 to 2.53$)$ & - \\
\hline \multicolumn{3}{|l|}{ Days after treatment: } \\
\hline $1-14$ & 3.38 (1.89 to 6.04$)$ & 2.20 (1.23 to 3.95$)$ \\
\hline $15-30$ & 0.78 (0.25 to 2.42$)$ & - \\
\hline $31-90$ & 0.89 (0.51 to 1.56$)$ & - \\
\hline $91-365$ & 1.08 (0.83 to 1.41$)$ & - \\
\hline $366-730$ & $1.05(0.82$ to 1.35$)$ & - \\
\hline $731-1095$ & 0.96 (0.73 to 1.25$)$ & - \\
\hline \multicolumn{3}{|l|}{ Arrhythmia } \\
\hline No in sample & 309 & 143 \\
\hline Median age (years) & 64.0 & 71.1 \\
\hline Men (\%) & $163(52.8)$ & $86(60.1)$ \\
\hline 14 days before treatment & 2.45 (0.77 to 7.80$)$ & - \\
\hline \multicolumn{3}{|l|}{ Days after treatment: } \\
\hline $1-14$ & 5.07 (2.19 to 11.72$)$ & 2.49 (1.09 to 5.69$)$ \\
\hline $15-30$ & 0.00 (0.00 to INF) & - \\
\hline $31-90$ & 1.94 (0.98 to 3.85$)$ & - \\
\hline $91-365$ & 1.61 (1.05 to 2.45$)$ & - \\
\hline $366-730$ & 1.07 (0.69 to 1.67$)$ & - \\
\hline $731-1095$ & $1.02(0.78$ to 1.86$)$ & - \\
\hline \multicolumn{3}{|l|}{ Stroke } \\
\hline No in sample & 2279 & 852 \\
\hline Median age (years) & 66.0 & 73.0 \\
\hline Men (\%) & $1295(56.8)$ & $480(56.3)$ \\
\hline 14 days before treatment & $0.36(0.13$ to 0.96$)$ & - \\
\hline \multicolumn{3}{|l|}{ Days after treatment: } \\
\hline $1-14$ & 1.49 (0.91 to 2.44$)$ & 1.04 (0.63 to 1.71$)$ \\
\hline $15-30$ & 1.25 (0.75 to 2.08$)$ & - \\
\hline $31-90$ & 1.21 (0.92 to 1.59$)$ & - \\
\hline $91-365$ & $1.06(0.91$ to 1.24$)$ & - \\
\hline $366-730$ & 0.94 (0.81 to 1.08$)$ & - \\
\hline 731-1095 & $1.14(0.98$ to 1.31$)$ & - \\
\hline
\end{tabular}

Days 1-14=current use; days 15-30=recent use; days 31-1095=past use.

${ }^{*}$ Age at study start date for self controlled case series and at event date for case crossover study.

Although some patients with $H$ pylori could have severe gastrointestinal bleeding that might precipitate myocardial infarction, ${ }^{24}$ it was unlikely to have affected the findings in this study. We only included patients receiving outpatient $H$ pylori eradication treatment containing clarithromycin who were unlikely to have severe gastrointestinal bleeding. Therefore, we concluded that the increase in cardiovascular risk short term was not attributable to the infection that prompted antibiotic treatment, but rather to the use of clarithromycin. In recent years, case reports have also suggested clarithromycin has proarrhythmic potency that leads to QT prolongation, torsades de pointes, ${ }^{25-27}$ and other arrhythmias. ${ }^{28}$ This hypothesis could be explained by blockade of the potassium channel and therefore result in a prolonged duration of the action potential and early after depolarisation. ${ }^{25}$ Such a mechanism would be consistent with a short increased risk of arrhythmia, in keeping with the findings of our cohort and self controlled case series analysis. Given the increased risk of
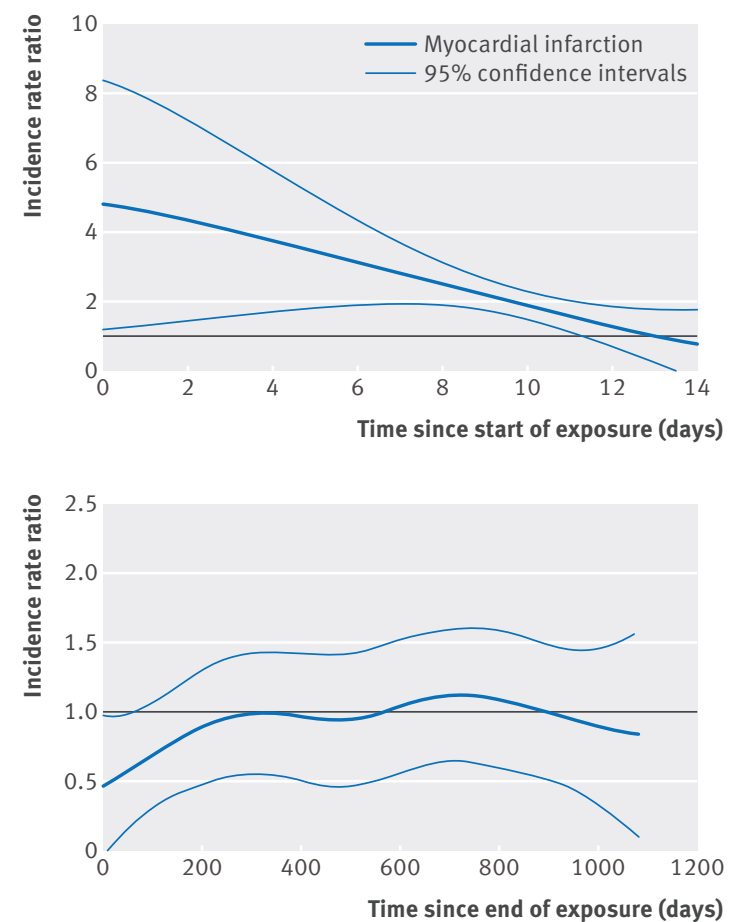

Fig 3 | Incidence rate ratio functions of myocardial infarction estimated by non-parametric self controlled case series method and $95 \%$ confidence bands

myocardial infarction found in our study, QT prolongation alone might not fully explain the cardiac effect, thus requiring further investigation. A recent case report suggested that clarithromycin might cause rupture of coronary plaque resulting from triggering of the allergic response. ${ }^{29}$ Possibly, arrhythmia may also have a role in plaque rupture leading to myocardial infarction.

A recent cohort study investigating the association between current use of clarithromycin and cardiac death in Denmark also reported a higher significant rate ratio of 1.76 (95\% confidence interval 1.08 to 2.85$){ }^{8}$ However, the study outcome was composite causes of death including, for example, myocardial infarction, arrhythmia, heart failure, and cardiomyopathy. Therefore, given the increased risk of composite causes of cardiac death, we cannot exclude the possibility that the cardiac effect may be explained by other mechanism pathways. Another observational Danish study reported no significant increase in the risk of a composite end point, including cardiovascular mortality and myocardial infarction among patients with ischaemic heart disease receiving $H$ pylori eradication treatment during a follow-up period of five years. ${ }^{30}$ In addition to cardiac mortality, we chose specific separate outcomes such as myocardial infarction and arrhythmia to investigate the cardiovascular effect associated with clarithromycin. In line with two Danish studies, our study showed that the risk of all cardiovascular outcomes of interest was increased during current use of clarithromycin, and no long term risk was observed. ${ }^{830}$

In contrast with our study, some observational studies reported cardiovascular risk associated with the use of clarithromycin long term, which is of concern 
because of its widespread use. A significantly higher risk of cardiovascular mortality was observed among the clarithromycin group in the CLARICOR (Effect of Clarithromycin on Mortality and Morbidity in Patients with Ischemic Heart Disease) trial during the follow-up period of 2.6 years, using public registers to ascertain outcomes. ${ }^{4}$ Notably, the agreement between hospital discharges and cause of death were $74 \%$ and $60 \%$, respectively, compared with formal adjudication. ${ }^{31}$ A recent cohort study by Svanstrom and colleagues also found that use of clarithromycin increased the risk of cardiovascular events in patients with acute exacerbations of chronic obstructive pulmonary disease and community acquired pneumonia over one year. ${ }^{9}$ In addition, the association between the use of clarithromycin and cardiovascular mortality was significant in patients with chronic obstructive pulmonary disease over one year. These two studies recruited patients with a high baseline cardiovascular risk, such as coronary heart diseases, chronic obstructive pulmonary disease, and community acquired pneumonia. This might reflect the fact that long term cardiovascular events and mortality associated with clarithromycin merely affect a selected population, as we could find no long term cardiovascular effect among the general population. Nevertheless, this might also reflect important underlying comorbidities between treatment groups that could not be fully addressed in those studies rather than a causal effect. Importantly, the study did not report non-cardiovascular mortality as a negative control outcome. ${ }^{9}$ Without this important information, the findings might be subject to important residual bias. We highly recommend that future studies should report control outcomes to detect residual confounding.

\section{Implications of this study}

The estimated absolute risk difference associated with current use of clarithromycin compared with amoxicillin in this study was 0.95 excess cardiac deaths per 1000 patients. Notably, the rate of cardiac mortality was 118.7 per 1000 person years in patients aged 75 or more receiving amoxicillin, considerably higher than that of patients aged 40-74 receiving amoxicillin (12.9 per 1000 person years). Moreover, we classified current use as treatment lasting for 14 days, which would also double the estimates of absolute risk difference when compared with classifying current use as a duration of seven days. As our study comprised patients aged 75 or more (35\% of our cohort) and used a longer follow-up period as current use, our estimate was noticeably higher than the one estimated by Svanstrom and colleagues ( 37 per million courses). ${ }^{8}$ In our subgroup analysis, 2.63 excess cardiac deaths and 5.77 excess myocardial infarction per 1000 patients were observed among patients aged 75 or more. In addition, absolute risk differences of cardiac death and myocardial infarction were also higher among patients with hypertensive diseases or diabetes mellitus. Before prescribing clarithromycin, the cardiovascular risk profile of individual patients, especially those aged 75 or more or with comorbidities, should be assessed carefully.

\section{Strengths and limitations of this study}

We used both a population based cohort study with a large cohort size, representative of the Hong Kong population, and a case only design, which renders the underlying differences between people less important. Accurate ascertainment of incident myocardial infarction and cardiac deaths was possible by linking data in the clinical data analysis and reporting system within primary, secondary, and tertiary healthcare services and death registries. The positive predictive value of myocardial infarction was high; our estimate was similar to most of the high quality validation studies on myocardial infarction. ${ }^{32}$ However, the current study has several limitations. Firstly, we did not include lifestyle risk factors such as smoking, alcohol consumption, and physical activity in the propensity score modelling. However, we introduced variables such as chronic obstructive pulmonary disease, diabetes, and other chronic diseases in the model that might partly represent the effects of these risk factors. Although the absence of this information may affect the performance of propensity score modelling in the cohort analysis, it is unlikely to significantly alter the findings on acute cardiovascular effects in the self controlled case series analysis. We were unable to obtain data on whether a drug was actually taken or when it was taken. Thus the true use of the drug is unknown and may result in some bias. In addition, details on cause of death were not available for some patients and therefore could have affected the cause specific analyses. This applied to about only $10 \%$ of deaths in this study, and thus would not have led to substantial bias. Moreover, only the cases from the Hong Kong west cluster were validated in this study. However, the coding practice is unlikely to differ between individual patients and between hospitals that are managed by the same statutory organisation. The positive predictive value of arrhythmia was relatively low, such that some of the outcomes we included may not have been true arrhythmias. This error would tend to underestimate any genuine causal association, assuming the likelihood of diagnostic inaccuracy was the same for both clarithromycin users and amoxicillin users. It may result from a low incidence of cardiac arrhythmia and less stringent diagnostic criteria when compared with myocardial infarction and stroke. Similarly, some studies also reported a wide range of positive predictive values of cardiac arrhythmia or cardiac arrest in different databases. ${ }^{33-35}$

\section{Conclusion}

In this study no long term cardiovascular risk was associated with clarithromycin but there was a suggestion of an increased short term risk of myocardial infarction, arrhythmia, and cardiac death associated with current use of clarithromycin among the Hong Kong population. Given the acute risk, clarithromycin should be prescribed with caution, especially to patients with a high baseline cardiovascular risk.

We thank the Hong Kong Hospital Authority for access to data and Michael Mok and Martijn Schuemie for their advice and contribution to our study.

Contributors: AYSW, AR, ID, and ICKW conceived and designed the study. AYSW and AR had principal responsibility for data analysis, drafting and revising the manuscript, and final approval. All authors 
contributed to the analysis, were responsible for the interpretation of the data, and drafted, revised, and gave final approval of the manuscript. AYSW and AR are the guarantors.

Funding: During the conduct of the study, ID was funded by grants from the Medical Research Council for this project. LS was funded by a grant from the Wellcome Trust. The sponsors had no role in the study design; collection, analysis, and interpretation of the data; the writing of the article; or the decision to submit the manuscript for publication. Competing interests: All authors have completed the ICMJE uniform disclosure form at www.icmje.org/coi_disclosure.pdf and declare: no financial relationships with any organisations that might have an interest in the submitted work in the previous three years; no other relationships or activities that could appear to have influenced the submitted work.

Ethical approval: This study was approved by the institutional review board of the University of Hong Kong/Hospital Authority Hong Kong west cluster (UW 14-032). Informed consent was not required for research based on routine data.

Data sharing: No additional data available.

Transparency: AYSW and AR affirm that this manuscript is an honest, accurate, and transparent account of the study being reported; that no important aspects of the study have been omitted; and that any discrepancies from the study as planned (and, if relevant, registered) have been explained.

This is an Open Access article distributed in accordance with the terms of the Creative Commons Attribution (CC BY 3.0) license, which permits others to distribute, remix, adapt and build upon this work, for commercial use, provided the original work is properly cited. See: http://creativecommons.org/licenses/by/3.0/.

1 Ho PL, Wong SY. Reducing bacterial resistance with IMPACT. 4th ed. 2012, Impact. www.chp.gov.hk/files/pdf/reducing_bacterial_ resistance_with_impact.pdf.

2 Zuckerman JM. Macrolides and ketolides: azithromycin, clarithromycin, telithromycin. Infect Dis Clin North Am 2004;18: 621 49, xi. doi:10.1016/j.idc.2004.04.01015308279

3 Sinisalo I. Mattila K, Valtonen VClarithromycin in Acute Coronary Syndrome Patients in Finland (CLARIFY) Study Group. Effect of 3 months of antimicrobial treatment with clarithromycin in acute non-q-wave coronary syndrome. Circulation 2002;105: 1555-60. doi:10.1161/01.CIR.0000012544.07696.1F11927522

4 Jespersen CM, Als-Nielsen B, Damgaard MCLARICOR Trial Group. Randomised placebo controlled multicentre trial to assess short term clarithromycin for patients with stable coronary heart disease: CLARICOR trial. BM/ 2006;332: 22-7. doi:10.1136/ bmj.38666.653600.55 16339220

5 Berg HF, Maraha B, Scheffer GJ. Treatment with clarithromycin prior to coronary artery bypass graft surgery does not prevent subsequent cardiac events. Clin Infect Dis 2005;40: 358-65 doi:10.1086/427111 15668857

6 Gluud C, Als-Nielsen B, Damgaard MCLARICOR Trial Group. Clarithromycin for 2 weeks for stable coronary heart disease: 6 -year follow-up of the CLARICOR randomized trial and updated metaanalysis of antibiotics for coronary heart disease. Cardiology 2008:111: 280-7. doi:1011159/00012899418451646

7 Winkel P, Hilden J, Hansen JFCLARICOR trial group. Clarithromycin for stable coronary heart disease increases all-cause and cardiovascular mortality and cerebrovascular morbidity over 10 years in the CLARICOR randomised, blinded clinical trial. Int J Cardiol 2015;182: 459-65. doi:10.1016/j.ijcard.2015.01.020 25602299

8 Svanström H, Pasternak B, Hviid A. Use of clarithromycin and roxithromycin and risk of cardiac death: cohort study. $B M$ 2014;349: g4930. doi:10.1136/bmj.g4930 25139799

9 Schembri S, Williamson PA, Short PM. Cardiovascular events after clarithromycin use in lower respiratory tract infections: analysis of two prospective cohort studies. BMJ 2013;346: f1235. doi:10.1136/bmj. f1235 23525864

$10 \mathrm{HAHO} /$ ITD. Clinical Data Analysis \& Reporting System (CDARS) user's manual. In: Hospital Authority, second ed. Hong Kong, 2003:3.

11 Chui CS, Man KK, Cheng CL. An investigation of the potential association between retinal detachment and oral fluoroquinolones: a self-controlled case series study. J Antimicrob Chemother 2014;69: 2563-7. doi:10.1093/jac/dku145 24833754

12 Man KK, Chan EW, Coghill D. Methylphenidate and the risk of trauma. Pediatrics 2015:135: 40-8 doi:10.1542/peds.2014-1738 25511122

13 Chiu SS, Lau YL, Chan KH, Wong WH, Peiris JS. Influenza-related hospitalizations among children in Hong Kong. N Engl J Med 2002:347: 2097-103, doi:10.1056/NEIMoa02054612501221
14 Whitaker HJ, Farrington CP, Spiessens B, Musonda P. Tutorial in biostatistics: the self-controlled case series method. Stat Med 2006;25: 1768-97. doi:10.1002/sim.230216220518

15 Smeeth L, Thomas SL, Hall AJ, Hubbard R, Farrington P, Vallance P. Risk of myocardial infarction and stroke after acute infection or vaccination. N Engl / Med 2004;351: 2611-8. doi:10.1056/ NEIMoa04174715602021

16 Ghebremichael-Weldeselassie Y, Whitaker H, Farrington C. Spline-based self-controlled case series method. 2015. http:// statistics.open.ac.uk/802576CB00593013/(httpInfoFiles)/ DE71C5F9EF48876E80257DEA0052905A/\$file/non-parametricSCCS.pdf.

17 Hospital Authority. Clinical services plan for the Hong Kong west cluster. 2015. www.ha.org.hk/upload/publication_44/453.pdf.

18 Stürmer T, Rothman KJ, Avorn J, Glynn RJ. Treatment effects in the presence of unmeasured confounding: dealing with observations in the tails of the propensity score distribution--a simulation study. Am J Epidemiol 2010;172: 843-54. doi:10.1093/aje/ kwq198 20716704

19 Stürmer T, Wyss R, Glynn RJ, Brookhart MA. Propensity scores for confounder adjustment when assessing the effects of medical interventions using nonexperimental study designs. J Intern Med 2014;275: 570-80. doi:10.1111/joim.12197 24520806

20 Ray WA, Murray KT, Hall K, Arbogast PG, Stein CM. Azithromycin and the risk of cardiovascular death. N Engl J Med 2012;366: 1881-90. doi:10.1056/NEJMoa1003833 22591294

21 Svanström H, Pasternak B. Hviid A. Use of azithromycin and death from cardiovascular causes. N Engl J Med 2013;368: 1704-12. doi:10.1056/NEJMoa1300799 23635050

22 Maclure M, Mittleman MA. Should we use a case-crossover design?Annu Rev Public Health 2000;21: 193-221. doi:10.1146/ annurev.publhealth.21.1.193 10884952

23 Douglas IJ, Evans SJ, Hingorani AD. Clopidogrel and interaction with proton pump inhibitors: comparison between cohort and within person study designs. BMJ 2012;345: e4388. doi:10.1136/bmj. e4388 22782731

24 Cappell MS. Gastrointestinal bleeding associated with myocardial infarction. Gastroenterol Clin North Am 2000;29: 423-44, vi. doi:10.1016/S0889-8553(05)70121-710836188

25 Lee KL, Jim MH, Tang SC, Tai YT. QT prolongation and Torsades de Pointes associated with clarithromycin. Am J Med 1998;104: 395-6. doi:10.1016/S0002-9343(98)00059-X 9576415

26 Hensey C, Keane D. Clarithromycin induced torsade de pointes. Ir J Med Sci 2008;177: 67-8. doi:10.1007/s11845-007-0057-317618400

27 Kamochi H, Nii T, Eguchi K. Clarithromycin associated with torsades de pointes. Jpn Circ J 1999;63: 421-2. doi:10.1253/jcj.63.421 10943628

28 Kundu S, Williams SR, Nordt SP, Clark RF. Clarithromycin-induced ventricular tachycardia. Ann Emerg Med 1997:30: 542-4. doi:10.1016/ S0196-0644(97)70019-2 932687

29 Bilginoglu M, Akyel A, Doğan M, Sunman H, Yeter E. Acute coronary syndrome secondary to clarithromycin: the first case and review of the literature. Turk Kardiyol Dern Ars 2014;42: 461-3. doi:10.5543/ tkda.2014.9289125080953

30 Andersen SS, Hansen ML, Norgaard ML. Clarithromycin use and risk of death in patients with ischemic heart disease. Cardiology 2010;116: 89-97. doi:10.1159/000315394 20523043

31 Kjoller E, Hilden J, Winkel P Agreement between public register and adjudication committee outcome in a cardiovascular randomized clinical trial.Am Heart/ 2014;168:197-204e1-4.

32 McCormick N, Lacaille D, Bhole V, Avina-Zubieta JA. Validity of myocardial infarction diagnoses in administrative databases: a systematic review. PLoS One 2014;9: e92286. doi:10.1371/journal. pone.009228624682186

33 Khan NF, Harrison SE, Rose PW. Validity of diagnostic coding within the General Practice Research Database: a systematic review. Br I Gen Pract 2010;60: 128-36. doi:10.3399/bjgp10X483562 20202356

34 Joensen AM, Jensen MK, Overvad K. Predictive values of acute coronary syndrome discharge diagnoses differed in the Danish National Patient Registry. / Clin Epidemiol 2009:62: 188-94. doi:10.1016/j.jclinepi.2008.03.005 18722087

35 Tamariz L, Harkins T, Nair V. A systematic review of validated methods for identifying ventricular arrhythmias using administrative and claims data. Pharmacoepidemiol Drug Saf 2012;21(Suppl 1): 148-53. doi:10.1002/pds.2340 22262601

(c) BMJ Publishing Group Ltd 2016

Appendix: Supplementary information on cardiovascular outcomes associated with use of clarithromycin 